$1989,38,1$

удК $550.93: 535.377$

Галина ХЮТТ, И. ЯЭК, Я. ЧОНКА

\title{
ФИЗИЧЕСКИЕ ОСНОВЫ ОПТИЧЕСКОГО МЕТОДА ДАТИРОВАНИЯ ОТЛОЖЕНИЙ
}

Настоящая работа является частью проекта «Разработка методов оптического датирования отложений» и касается палеодозиметрических аспектов фотостимулированной (ФСЛ) люминесценции К-полевых шпатов.

Стимулом к исследованию послужили работы канадских и английских ученых (Huntley и др., 1985; Aitken и др., 1986), а также собственные исследования по ФСЛ сульфида кальция (Яэк и др., 1969; Хютт, 1972). В качестве объекта исследования были выбраны К-полевые шпаты в связи с их высокой чувствительностью к свету и достаточно интенсивной вспышкой при термолюминесцентном (ТЛ) датировании.

\section{Аппаратура}

В принципе для таких исследований нужна система из двух монохроматоров и источник света, по спектральному составу близкий к естественному. В качестве источника света нами была использована Хе-лампа, а монохроматор, выделяющий излучение, был заменен светофильтрами, пропускающими излучение $\simeq 400$ нм $(3,1$ эВ) и подавляющими стимулирующую радиацию: практически фон отсутствовал во всей спектральной области исследования.

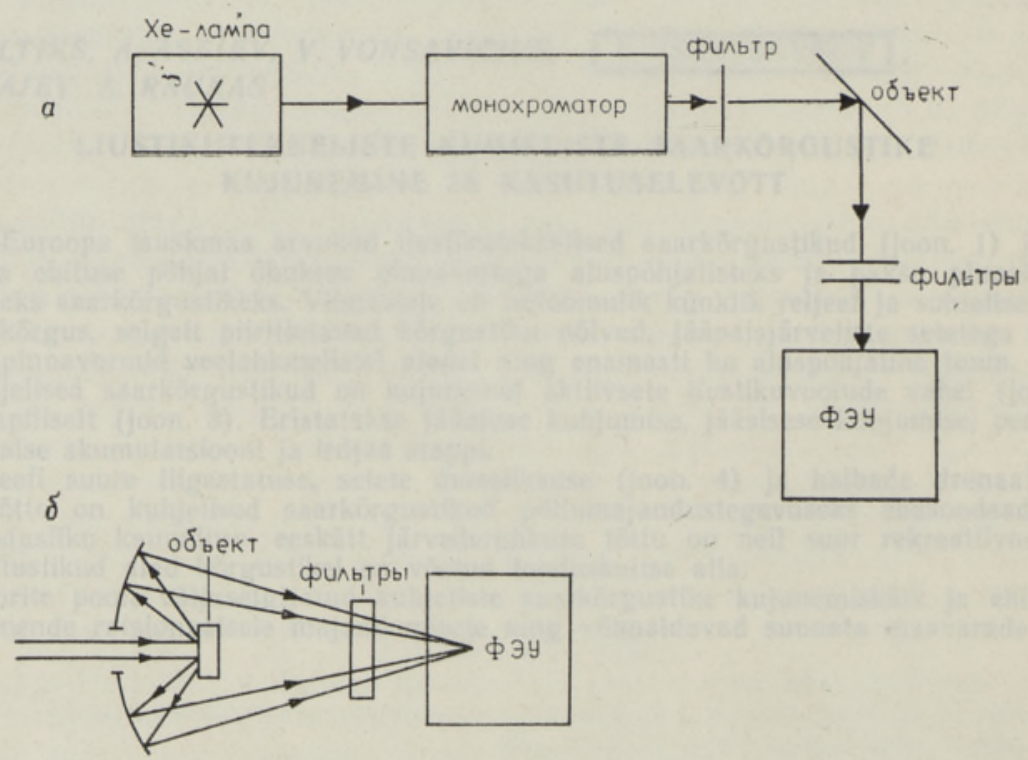

Рис, 1. Схема исследовательской установки (а) и камеры (б). 
В связи с тем, что концентрация центров окраски не очень велика, необходимо было обеспечить высокую эффективность светосбора при регистрации вспышки. Была использована специальная камера с эллиптическим зеркалом, в одном фокусе которого находится исследуемый объект, а в другом - катод ФЭУ (рис. 1, $a, б)$. В камере возможно осуществлять нагрев образцов от -80 до $+400^{\circ} \mathrm{C}$. Система обеспечивает надежное измерение спектров стимуляции оптической вспышки в интервале энергий 2,25-0,83 эВ (500-1500 нм).

\section{Спектры стимуляции оптической вспышки}

Были исследованы спектры стимуляции - зависимость интенсивности вспышки от длины волны воздействующего света - минерала микроклина, кривая ТЛ которого аналогична таковой для К-полевых шпатов из отложений, а также К-полевых шпатов, экстрагированных из отложений Северо-Западного региона СССР. В эксперименте использовались естественные, а также облученные рентгеновскими лучами образцы (рис. $2,1-3$ ). В спектре стимуляции, поправленном на распределение лампы, можно выделить ряд максимумов 2,$25 ; 1,43 ; 1,33$ и 1,29 эВ. Последний наблюдается лишь в свежеоблученных образцах.

Первым условием пригодности соответствующих уровней захвата для датирования является достаточная стабильность запасенной на них оптической светосуммы, что возможно установить лишь исследованием их связи с соответствующими максимумами термостимулированной люминесценции (ТСЛ). При этом нет необходимости следить за остаточной светосуммой в отдельных максимумах ТСЛ при воздействии на

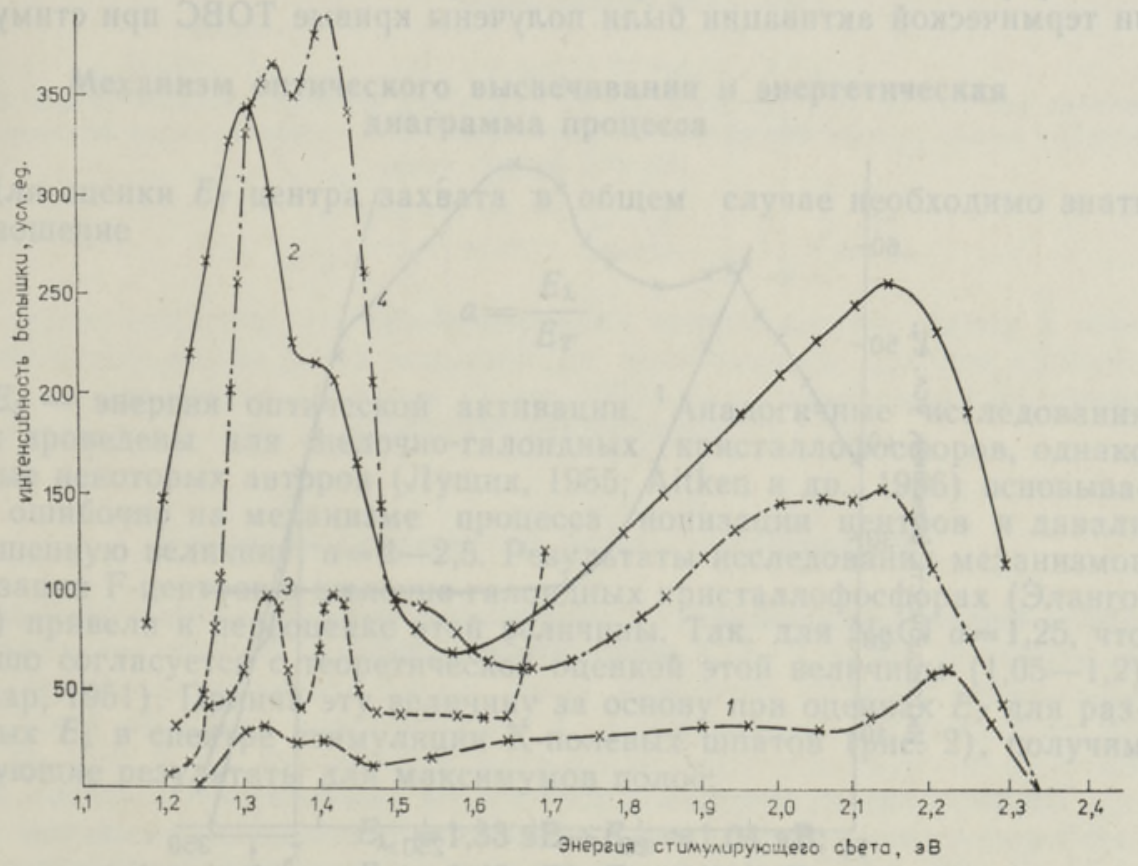

Рис. 2. Спектры стимуляции оптической вспышки К-полевого шпата: 1 - естественный образец; 2 - облученный рентгеном микроклин; 3 - облученный рентгеном естественный образец; 4 - облученный рентгеном и прогретый до $200^{\circ} \mathrm{C}$ образец. Спектры поправлены на распределение лампы. При регистрации выделяется полоса свечения 3,1 эВ. 
объект различными длинами волн, так как при оптическом датировании предполагается использовать лишь наиболее светочувствительные ловушки, т.е. те, которые обуславливают быструю компоненту кривой, описывающей кинетику высвечивания палеодозиметров (Wintle, Huntley, 1982).

Существует другой известный способ поисков корреляции фотостимулированной люминесценции (ФСЛ) с ТСЛ: исследование зависимости интенсивности вспышки от температуры, т. е. измерение кривых термооптического высвечивания (ТОВС), а также измерение спектров стимуляции с предварительным прогревом облученного образца до различных температур.

\section{Корреляция ФСЛ и ТСЛ}

В спектре стимуляции естественного и облученного с предварительным прогревом до $200^{\circ} \mathrm{C}$ образцов наблюдаются одни и те же полосы (рис. 2). Обращает на себя внимание высокая термическая стабильность «инфракрасных» ловушек, - ожидалось, что информация, запасенная на столь мелких ловушках не должна сохраняться. Также видно, что область 2,0 - 2,3 эВ явно неэлементарна, некоторое же смещение высокоэнергетического максимума $\simeq 2,25$ эВ, скорее всего, связано с тем, что выделяющий излучение светофильтр обрезает спектр и мы фиксируем лишь «хвост» более высокоэнергетического компонента. Итак, вся область спектра от 1,25 до 2,35 эВ обусловлена термически достаточно стабильными уровнями захвата.

В спектрах стимуляции оптической вспышки по оси абсцисс отложена энергия оптической активации центров - она, как правило, больше энергии термической активации, определяющей стабильность запасенной информации на соответствующих ловушках. Для определения энергии термической активации были получены кривые ТОВС при стимуля-

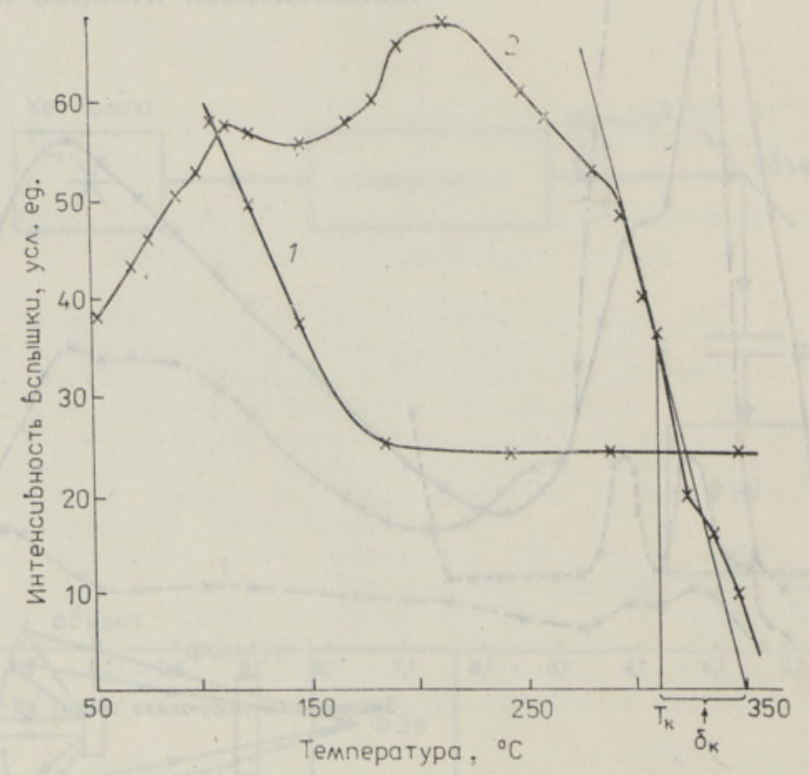

Рис. 3. Термооптическое высвечивание при стимуляции светом в полосах: $1-2,25$ эВ; $2-1,43$ эВ. 
ции в максимумах высокоэнергетической и низкоэнергетической частей спектра стимуляции. Возможны два варианта получения кривой ТОВС: а) измерение вспышки при постоянной температуре в режиме пилообразного нагрева: вспышка измеряется, например, при $80^{\circ} \mathrm{C}$ после каждого нагрева до все более высокой температуры с последующим охлаждением до $80^{\circ} \mathrm{C}$. В таком режиме было измерено поведение вспышки при стимуляции в области 2,25 эВ. Вспышка остается стабильной вплоть до $350{ }^{\circ} \mathrm{C}$ (рис. 3,1 ); б) наиболее прямой способ оценки энергии термической активации связан с ТОВС, при котором интенсивность вспышки измеряется через определенные температурные интервалы на фоне кривой ТСЛ в режиме равномерного нагрева. Таким образом были получены кривые ТОВС при стимуляции в полосах 1,43 и 2,25 эВ (рис. 3,2 ).

Согласно теории метода, разработанной Ч. Б. Лущиком (1955), термическая стабильность соответствующего дефекта определяется последним высокотемпературным спадом ТОВС. Термическая энергия активации $\left(E_{T}\right)$ соответствующего центра по кривой ТОВС определяется по формуле (І порядок кинетики)

$$
E_{T}=\frac{2 k T_{k}^{2}}{\delta_{k}},
$$

где $k$ - константа Больцмана, $T_{h}$ - температура полуспада интенсивности сигнала $\left(320^{\circ} \mathrm{C}\right), \delta_{k}-$ температурный интервал реализации этого полуспада $\left(330^{\circ} \mathrm{C}\right)$.

Оцененная таким образом $E_{T}$ соответствующего дефекта составляет

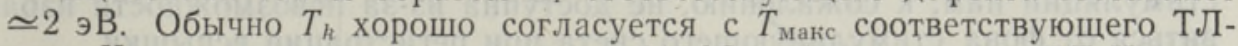
пика. Как известно, именно в области $310^{\circ} \mathrm{C}$ наблюдается пик ТЛ К-полевых шпатов, таким образом, можно полагать, что ФСЛ и ТСЛ обусловлены одним и тем же дефектом.

\title{
Механизм оптического высвечивания и энергетическая диаграмма процесса
}

\author{
Для оценки $E_{T}$ центра захвата в общем случае необходимо знать \\ соотношение

$$
a=\frac{E_{\lambda}}{E_{T}},
$$

где $E_{\lambda}$ - энергия оптической активации. Аналогичные исследования были проведены для щелочно-галоидных кристаллофосфоров, однако данные некоторых авторов (Лущик, 1955; Aitken и др., 1986) основывались ошибочно на механизме процесса ионизации центров и давали завышенную величину: $a=2-2,5$. Результаты исследования механизмов ионизации F-центров в щелочно-галоидных кристаллофосфорах (Эланго, 1960) привели к переоценке этой величины. Так, для $\mathrm{NaCl} a=1,25$, что хорошо согласуется с теоретической оценкой этой величины $(1,05-1,2)$ (Пекар, 1951). Приняв эту величину за основу при оценках $E_{T}$ для различных $E_{\lambda}$ в спектре стимуляции К-полевых шпатов (рис. 2), получим следующие результаты для максимумов полос:

$$
\begin{aligned}
& E_{\lambda_{1}}=1,33 \text { э } \rightarrow E_{T_{1}} \simeq 1,04 \text { э } \mathrm{B} ; \\
& E_{\lambda_{2}}=1,43 \text { э } \rightarrow E_{T_{2}} \simeq 1,12 \text { э } ; \\
& E_{\lambda_{3}}=2,25 \text { э } \rightarrow E_{T_{3}} \simeq 1,8 \text { э } \mathrm{B} .
\end{aligned}
$$

Для объяснения возникшего противоречия между небольшой энергией термической активации «инфракрасных» центров и их фактической 
термической стабильностью необходимо проанализировать механизм процесса оптической вспышки.

Рост интенсивности вспышки в некоторых стадиях нагрева может быть обусловлен либо перераспределением дырок на центрах рекомбинации, либо фототермическим характером освобождения носителей заряда с ловушек (рис. 3, 2). Разделение этих возможностей легко осуществимо предварительным нагревом образца выше температуры роста вспышки. В случае перераспределения дырок рост устраняется, в случае же фототермического механизма - нет. Для обоих полос с $E_{\lambda}=1,43$ и 1,33 эВ характерен фототермический процесс ионизации, аналогичный ситуации, реализуемой при стимуляции F-светом в ЩГК. Согласно предлагаемой схеме рекомбинационного процесса с помощью инфракрасного света происходит лишь заброс носителей с основного состояния $\left(E_{T} \simeq 2,0\right.$ эВ) на возбужденный $\left(E_{T} \simeq 1,09 ; 1,16\right.$ эВ), а оттуда за счет дополнительной $E_{T} \simeq 0,8$ эВ $(2,0-1,2=0,8$ эВ) носители попадают в зону проводимости и рекомбинируют с центрами свечения (рис. 4). Поскольку возбужденные состояния расположены достаточно близко к зоне проводимости, то уже при комнатной температуре вспышка, стимулируемая инфракрасным светом, достаточно велика за счет моментального фединга с возбужденных состояний. Однако небольшой нагрев до $40-50^{\circ} \mathrm{C}$ приводит к увеличению вспышки в 3-4 раза (вероятность освобождения носителей с уровней захвата экспоненциально зависит от температуры). По нашему мнению, оба возбужденные состояния соответствуют одному основному состоянию, оптическая энергия активации которого составляет 2,5 эВ $\left(E_{\lambda} \simeq E_{T} \cdot 1,25=2,0\right.$ эВ $\cdot 1,25=2,5$ эВ $)$. Эта область оптической стимуляции нам, к сожалению, не доступна из-за невозможности избавиться от фона стимулирующего света при регистрации вспышки.
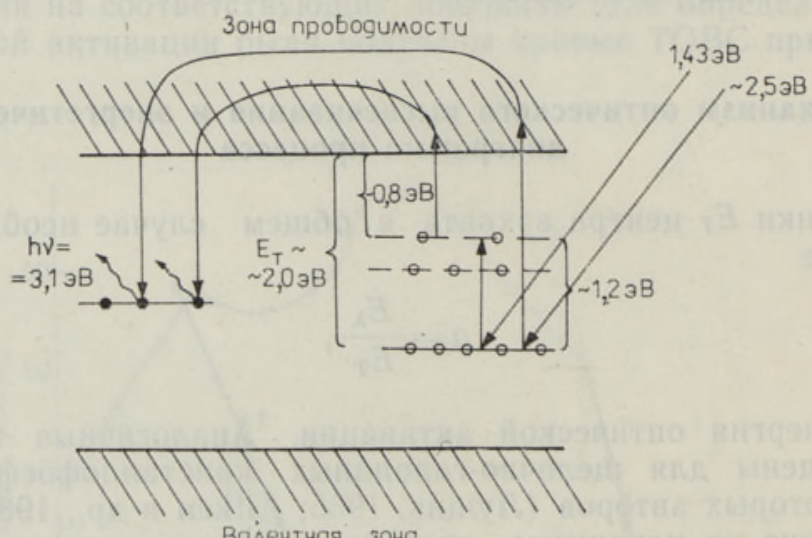

Ваментная зона

Рис. 4. Энергетическая диаграмма и схема рекомбинационного процесса фотостимулированной люминесценции.

Таким образом, ловушка с $E_{T} \simeq 2,0$ эВ высвечивается светом 2,5 эВ (496 нм) в результате прямой оптической ионизации и светом 1,43 и 1,33 эВ (866 и 932 нм) в результате термооптического механизма ионизации.

Была предпринята попытка исследования прямой связи зеленой области спектра с инфракрасной. Измерялись спектры высвечивающего действия, - при этом определялось влияние стимуляции в области 
полосы с максимумом 2,25 эВ на интенсивность вспышки 1,33 эВ. Проявились весьма сложные зависимости, связанные с перераспределением носителей. При этом интенсивность вспышки при стимуляции инфракрасным светом зависит от времени высвечивания зеленым (рис. 5). Спад интенсивности практически до нуля обнадеживает с точки зрения реализации нуль-момента для «инфракрасных» центров в процессе формирования отложений в результате солнечной экспозиции. Облучение дневным светом в течение $1-2$ мин полностью уничтожает вспышку во всей спектральной области стимуляции.

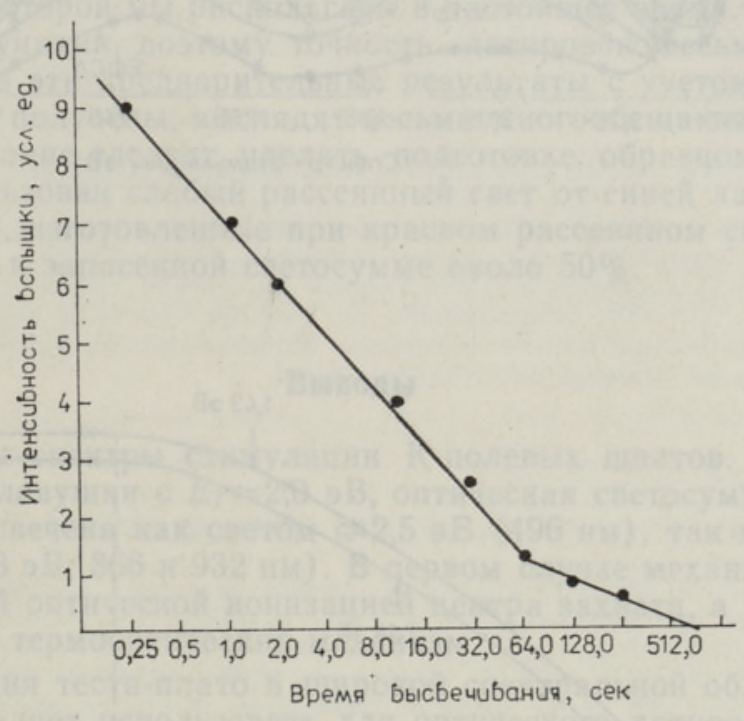

Рис. 5. Высвечивающее действие света 2,25 эВ на вспышку 1,43 эВ (на оси ординат интенсивность вспышки прн стимуляции светом 1,43 эВ; на оси абсцисс время высвечивания светом 2,25 эВ).

Следует отметить, что при стимуляции инфракрасным светом в течение темновой паузы роста вспышки не наблюдалось. Данное явление, известное для многих объектов (Хютт, 1972), благоприятствует практическому использованию этой области стимуляции.

\section{Палеодозиметрический аспект исследований}

Факт достаточно высокой термической стабильности ловушек, обусловливающих полосы стимуляции в области 2,14-1,30 эВ, подтверждается своеобразным тестом-плато: были измерены спектры стимуляции оптической вспышки естественного и $\gamma$-облученного образцов до доз 1500 Гр (рис. 6, a). Реализация теста-плато свидетельствует об однотипности палеодозиметрических параметров соответствующих уровней захвата. Аналогичная картина наблюдается и при дозах стимуляции 1,43 и 2,14 эВ (рис. $6, б)$.

Ориентировочная возможность оценки времени геологических событий составляет 500-600 тыс. лет. Была предпринята попытка предварительного датирования некоторых образцов, возраст которых был известен на основе других методов (таблица). 


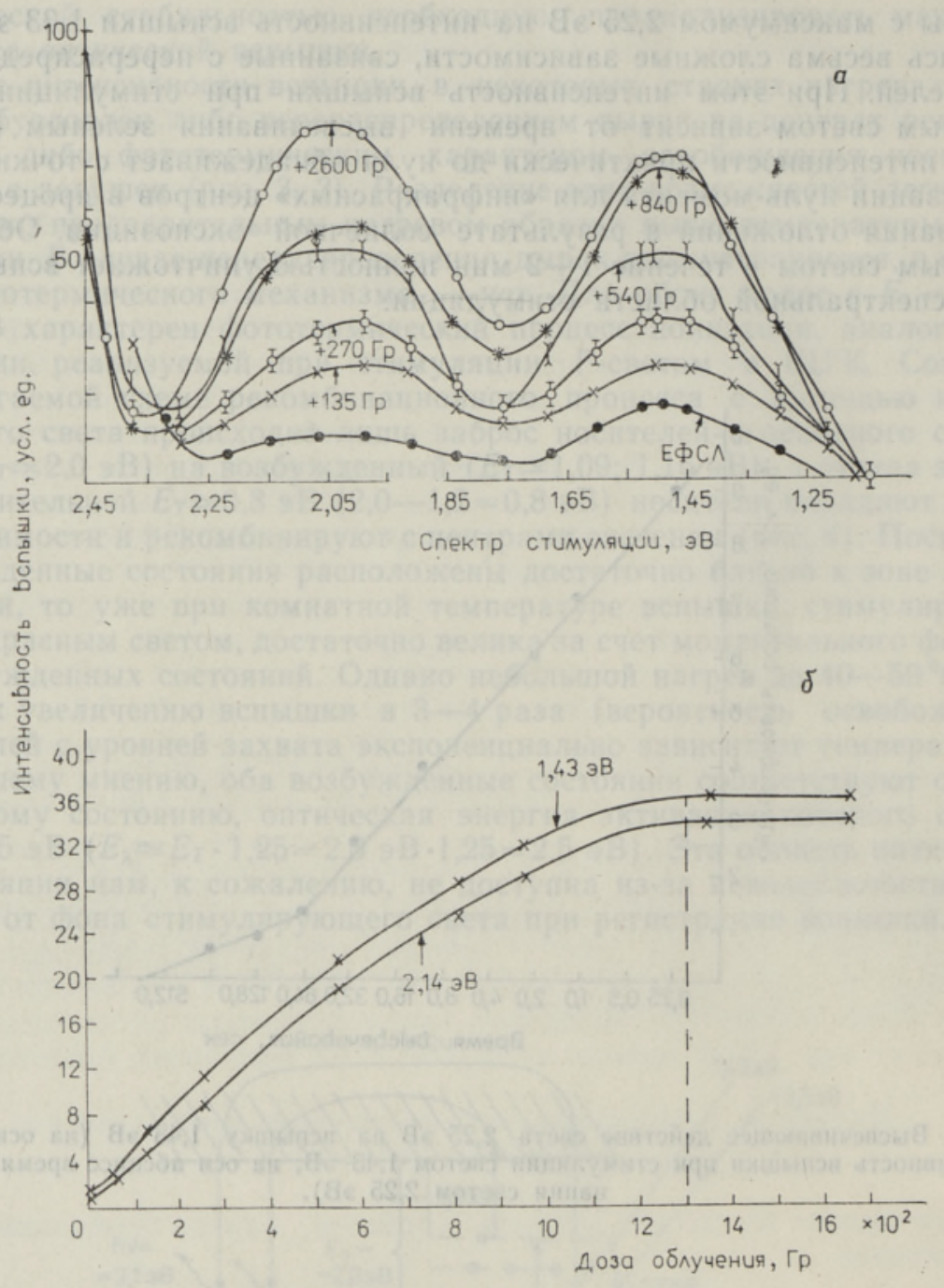

Рис. 6. $a$ - спектры стимуляции естественного образца К-полевого шпата с $\beta$-дозой облучения (тест-плато). Спектры не поправлены на распределение лампы, выделяется полоса свечения $\simeq 3,1$ эВ (ЕФСЛ - естественная фотостимулированная люминесценция); б - зависимость интенсивности вспышки от дозы при стимуляции светом 1,43 и 2,14 эВ.

\section{Сравнение результатов оптического датирования} с другими методами $(E=1,43$ эВ $)$, тыс. лет

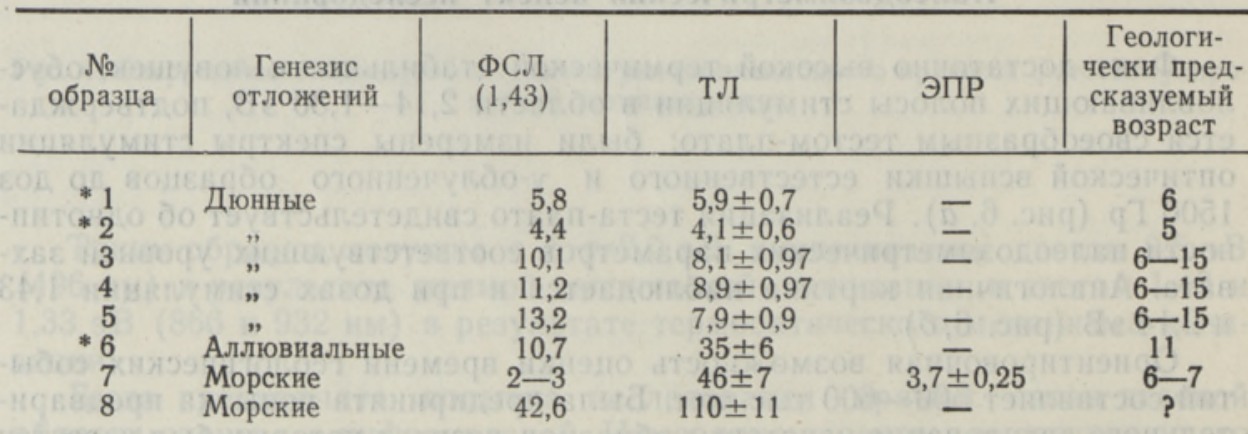

* Оптическое датирование осуществлялось стимуляцией светом 1,43 и 2,14 эВ. 
ТЛ-датирование эоловых образцов проводили по предложенной А. К. Сингхви методике полного высвечивания (Singhvi и др., 1982). Для первых двух образцов гипотеза полного высвечивания, по-видимому, близка к реальности, отсюда хорошее совпадение результатов ТЛ- и оптического датирования. Для дюнных образцов $3-5$ (таблица) ТЛ-результаты не соответствовали стратиграфии, - оптическое датирование проявило зависимость возраста от глубины отбора. Наиболее интересен результат датирования аллювиальных отложений ТЛ-методом, при котором был получен возраст, резко противоречащий геологической картине, в то время как оптическая датировка очень удачно соответствовала ей.

Установка, которой мы располагаем в настоящее время, имеет исследовательские функции, поэтому точность датировок весьма проблематична. Однако и эти предварительные результаты с учетом той простоты, с какой они получены, выглядят весьма многообещающими.

Особое внимание следует уделять подготовке образцов к датированию. Был использован слабый рассеянный свет от синей лампы накаливания. Образцы, изготовленные при красном рассеянном свете, обнаруживали потерю в запасенной светосумме около $50 \%$.

\section{Выводы}

1. Измерены спектры стимуляции К-полевых шпатов. Установлено существование ловушки с $E_{T} \simeq 2,0$ эВ, оптическая светосумма с которой может быть высвечена как светом $\simeq 2,5$ эВ (496 нм), так и инфракрасным $(1,43$ и 1,33 эВ; 866 и 932 нм). В первом случае механизм процесса связан с прямой оптической ионизацией центра захвата, а во втором осуществляется термооптический механизм.

2. Реализация теста-плато в широкой спектральной области от 2,25 до 1,3 эВ позволяет использовать для оптического датирования любой источник света из этого энергетического интервала. Наиболее простой и удобной в пользовании, по-видимому, является лампа накаливания, в спектре которой достаточно хорошо представлена инфракрасная область. Удобство использования инфракрасного света состоит и в том, что возможно вообще не использовать монохроматор, заменив его светофильтрами, так как излучение (400 нм) и стимуляция (900 нм) достаточно далеки друг от друга, что снимает проблему фона при регистрации сигнала.

3. Ориентировочный возрастной интервал оптического датирования геологических событий составляет $2 \cdot 10^{3}-6 \cdot 10^{5}$ лет.

4. В методике оптического датирования К-полевых шпатов основой является не селекция светочувствительных зерен, а существование глубокого $\left(E_{T} \cong 2,0\right.$ эВ) уровня захвата, оптическая светосумма с которого достаточно легко высвечивается естественным светом, что и обеспечивает «нулирование» в процессе формирования отложений.

\section{ЛИТЕРАТУРА}

Лущик Ч. Б. Исследование центров захвата в щелочно-галоидных кристаллофосфорах // Тр. ИФА, 1955, № 3.

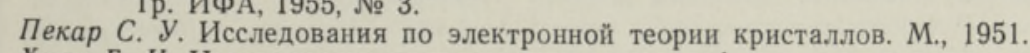

Хютт Г. И. Исследование природы примесных дефектов легированного сульфида кальция. Канд. дис. Тарту, 1972.

Эланго М. А. Исследование термического разрушения F-центров монокристаллов $\mathrm{NaCl}$ // Тр. ИФА, 1960, № 12, 197-225. 
Яэк И. В., Лайсаар Т. И., Раудсепn Р. Н., Хютт Г. И. Фото- и термостимулированная люминесценция CaS-фосфоров // Тр. ИФА, 1969, № 35, 88-104.

Aitken, M. J., Rhodes, E. J., Robinson, P. D., Smith, B. W., Templer, R. H. Optical dating: progress report // Methodology of TL-dating. Abstr. Int. TL-workshop. Tallinn, 1986.

Huntley, D. J., Godfrey-Smith, D. I., Thewalt, M. L. W. Optical dating of sediments // Nature (Lond.), 1985, 313, 105-107.

Singhvi, A. K., Sharma, Y. P., Agrawal, D. P. Thermoluminescence dating of sand dunes in Rajastan // Nature (India), 1982, 295, 313-315.

Wintle, A. G., Huntley, D. J. Thermoluminescence dating of sediments // Quat. Sci. Rev., $1982, \mathrm{~N} 1,31-53$.

Ннститут геологии

Академии наук Эстонской ССР
Поступила в редакцию

24/III 1988

\section{Galina HOTT, I. JAEK, J. TSONKA}

\section{SETETE DATEERIMISE OPTILISE MEETODI FUUSIKALISED ALUSED}

On uuritud kvartspōldpao optilise sähvatuse stimulatsioonispektreid ja näidatud suhteliselt sügava valgustundliku haardenivoo olemasolu $(\simeq 2 \mathrm{eV})$. Sellel salvestunud valgussumma võib välja kiirata nii nähtava valguse $(\simeq 2,5 \mathrm{eV})$ kui ka infrapunase valguse piirkonnas $(1,43 ; 1,33 \mathrm{eV})$. Diagrammidel on näidatud protsessi mehhanismi ja energeetikat. On välja pakutud optilise dateerimise lihtne seade, mis töötab infrapunase valgusega, ning esitatud esialgsed dateerimistulemused.

\section{Galina HOTT, I. JAEK, Y. TSHONKA}

\section{PHYSICAL PRINCIPLES OF AN OPTICAL DATING METHOD OF SEDIMENTS}

K-feldspars optical response stimulation spectra have been studied. The presence of a sufficiently deep light-sensitive trap $(\simeq 2 \mathrm{eV})$ is shown. The optical light sum, stored on it may be bleached both as a visible $(\simeq 2.5 \mathrm{eV})$ and infrared $(1.43 ; 1.33 \mathrm{eV})$ light region. Energetical diagram and the mechanism of the process is presented. A simple variant of optical dating device using infrared light is offered and some preliminary dating results disĉussed. 